\title{
Ultrasmall AGulX theranostic nanoparticles for vascular-targeted interstitial photodynamic therapy of glioblastoma
}

This article was published in the following Dove Press journal:

International Journal of Nanomedicine

26 September 2017

Number of times this article has been viewed

\author{
Eloïse Thomas' \\ Ludovic Colombeau ${ }^{2}$ \\ Mickaël Gries ${ }^{3,4}$ \\ Thibaut Peterlini ${ }^{3,4}$ \\ Clélia Mathieu' \\ Noémie Thomas ${ }^{3,4}$ \\ Cédric Boura ${ }^{3,4}$ \\ Céline Frochot ${ }^{2}$ \\ Régis Vanderesse ${ }^{5}$ \\ François Lux' \\ Muriel Barberi-Heyob 3,4 \\ Olivier Tillement ${ }^{\prime}$
}

'Université Lyon, Université Claude Bernard Lyon I, Centre National de la Recherche Scientifique (CNRS), Institut Lumière Matière, Lyon,

${ }^{2}$ Laboratoire Réactions et Génie des Procédés, Université de LorraineCNRS, Nancy, ${ }^{3}$ Université de Lorraine, Research Center for Automatic Control of Nancy (CRAN), ${ }^{4}$ CNRS, CRAN, Vandœuvre-lès-Nancy, ${ }^{5}$ Laboratoire de Chimie Physique Macromoléculaire, Université de Lorraine-CNRS, Nancy, France

Correspondence: François Lux Université Lyon, Université Claude Bernard Lyon I, CNRS, Institut Lumière Matière, F-69622 Lyon, France Email francois.lux@univ-lyonI.fr
Abstract: Despite combined treatments, glioblastoma outcome remains poor with frequent local recurrences, indicating that a more efficient and local therapy is needed. In this way, vascular-targeted photodynamic therapy (VTP) could help tumor eradication by destroying its neovessels. In this study, we designed a polysiloxane-based nanoparticle (NP) combining a magnetic resonance imaging (MRI) contrast agent, a photosensitizer (PS) and a new ligand peptide motif (KDKPPR) targeting neuropilin-1 (NRP-1), a receptor overexpressed by angiogenic endothelial cells of the tumor vasculature. This structure achieves the detection of the tumor tissue and its proliferating part by MRI analysis, followed by its treatment by VTP. The photophysical properties of the PS and the peptide affinity for NRP-1 recombinant protein were preserved after the functionalization of NPs. Cellular uptake of NPs by human umbilical vein endothelial cells (HUVEC) was increased twice compared to NPs without the KDKPPR peptide moiety or conjugated with a scramble peptide. NPs induced no cytotoxicity without light exposure but conferred a photocytotoxic effect to cells after photodynamic therapy (PDT). The in vivo selectivity, evaluated using a skinfold chamber model in mice, confirms that the functionalized NPs with KDKPPR peptide moiety were localized in the tumor vessel wall.

Keywords: nanoparticles, PDT, vascular targeting strategy, brain tumor, NRP-1, peptide ligand, MRI

\section{Introduction}

Despite multiple treatments, glioblastoma multiforme (GBM) remains the most frequent and malignant brain tumor with an overall survival of only 14.6 months. ${ }^{1}$ Currently, treatments often combine surgery, radiotherapy and chemotherapy, but a wide majority of GBM recurs locally, upwards of $80 \%$ of cases, leading to patient's death. ${ }^{2}$ This indicates that a more aggressive local treatment is needed to improve the local control. However, resection can hardly be a complete and curative intervention because of the migration of tumor cells into the healthy brain parenchyma. ${ }^{1}$ Furthermore, radiotherapy often induces side effects, impacting health and quality of life. In this context, photodynamic therapy (PDT) appears to be an interesting complementary approach in order to prevent GBM local recurrence and to improve local control with minimal side effects. ${ }^{2}$ PDT is based on the activation of a photosensitizer (PS) by light at an appropriate wavelength to generate reactive oxygen species, especially singlet oxygen. ${ }^{3}$ Besides, the radius of interaction for singlet oxygen is inferior to $0.2 \mu \mathrm{m}$ and the half life is short, from $0.03 \mu \mathrm{s}$ to $0.18 \mathrm{~ms}$, according to the medium, inducing cell damages in close proximity of the activated PS. ${ }^{4}$ Owing to the localization of the optical fiber into the tumor tissue, an interstitial PDT (iPDT) can be suggested. ${ }^{5}$ 
Several clinical trials have highlighted the interest of PDT for GBM. ${ }^{2,6-8}$ For Phase II clinical studies, we can mention the one of the Muragaki group on patients with primary malignant brain tumors including $13 \mathrm{GBM}$ cases $^{9}$ or the one of the Whelan group (NCT01966809) for recurrent high-grade gliomas using intraoperative PDT to eradicate cancer cells after resection as local recurrences in the surgical bed after tumor resection may be due to residual tumor cells.

The choice of the PS molecule is crucial to generate a photodynamic efficiency. Whereas many PSs have been developed, some issues remain and have to be overcome. ${ }^{10}$ First, numerous PSs are porphyrin derivatives and therefore poorly soluble in water; they can aggregate in physiological media, leading to a drastic decrease in singlet oxygen production and difficulties for intravenous injection. Moreover, they often display a low tumor tissue selectivity in vivo. ${ }^{11,12}$ During the last few decades, one solution emerged using multifunctional nanoparticles (NPs) for PDT, highlighting several advantages. ${ }^{13}$ The first one comes from the tumor selectivity. They can indeed specifically accumulate into the tumor tissue due to the enhanced permeability and retention (EPR) effect. Moreover, their surface can be functionalized to target a specific receptor overexpressed by neoplastic or angiogenic endothelial cells. ${ }^{14,15}$ Their composition can be modulated to protect the PS molecules, increasing their solubility in biological media, optimizing their lifetime in plasma or avoiding enzymatic degradation. Finally, by adding a contrast agent to the NPs, they can also be suggested at the same time for a treatment guided by imaging. It is therefore possible to detect the tumor, to plan the treatment (for instance, an appropriate PDT optical fiber positioned into the tumor volume), to optimize the drug-light interval before irradiation and to follow the tumor response by diffusion MRI. ${ }^{16}$

Different kinds of NPs have already been described for PDT. ${ }^{17-23}$ The ones based on polysiloxane are particularly interesting because their size and shape can be controlled, are biocompatible and can be easily functionalized. ${ }^{24} \mathrm{PS}$ molecules can be encapsulated inside pores of mesoporous NPs or grafted on the surface of a polysiloxane core. With this last strategy, singlet oxygen does not need to diffuse out of the NPs and it avoids the aggregation of PS molecules inside the pores. Our group recently described such kinds of NPs for iPDT applied to GBM. ${ }^{25}$ In this case, 5-(4-carboxyphenyl)10,15,20-triphenylporphyrin was grafted on the surface of an ultrasmall NP called AGuIX. AGuIX is composed of a polysiloxane core surrounded by DOTA@ $\mathrm{Gd}^{3+}$ complexes (DOTA, 1,4,7,10-tetraazacyclododecane-1,4,7,10-tetraacetic acid), enabling detection of NPs by magnetic resonance imaging (MRI). AGuIX@PS NPs were injected intravenously to nude rats bearing U87 intracranial GBM. Their tumor tissue accumulation was followed by MRI, and the optical fiber was precisely inserted into the tumor burden. Using AGuIX@PS, iPDT induced a statistically significantly therapeutic effect compared to control groups. It was also possible to determine early predictive markers by proton magnetic resonance (MR) spectroscopy and diffusion MRI to discriminate between nonresponding and responding animals. ${ }^{25}$

The indirect effects of PDT, including immune response and vascular destruction, are well known to play a major role in photodynamic efficiency. ${ }^{26-28}$ The latter effect may indirectly initiate a privation of nutrients and molecular oxygen into the tumor tissue. To asphyxiate some vascularized tumors, mainly the infiltrating part, a targeting strategy of the tumor vasculature may potentiate this effect. We also previously demonstrated that this effect would play a major role in the destruction of GBM by PDT. ${ }^{11,29}$ The goal of the present study was to synthesize an ultrasmall theranostic NP, presenting high affinity for neovessels. In addition to a better PDT efficiency, we can also expect to reach longer intra-tumor retention and detection by MRI of the real tumor volume, taking into account the proliferating part of the tumor tissue. Overexpressed receptors specifically located on angiogenic endothelial cells are therefore promising molecular target for PDT. Neuropilin-1 (NRP-1) is one of them; it is a receptor for vascular endothelial growth factor, isoforms A-165 and A-145 (VEGF-A ${ }_{165}$ and VEGF-A ${ }_{145}$ ), and is overexpressed in several cancers. ${ }^{30}$ The use of a chlorin conjugated to a heptapeptide (ATWLPPR) and their grafting on a NP has already been described for vascular-targeted photodynamic therapy (VTP) and has highlighted very promising in vitro and in vivo results..$^{11,26,29,31-33}$ Recently, we evidenced that new peptides have an improved affinity for NRP-1; DKPPR is one of them. ${ }^{34}$

This article focuses on the design of a new AGuIX-type nanoplatform for VTP using this DKPPR peptide moiety. For our research approach, a lysine $(\mathrm{K})$ had to be added at the $N$-terminal side of the DKPPR peptide. Subsequently, the KDKPPR peptide was coupled with a PS, 5-(4-carboxyphenyl)10,15,20-triphenylporphyrin, to the amine function of the lysine lateral side chain, producing PS@KDKPPR. This conjugate was then used in the design of this new nanoplatform for VTP by grafting at the surface of AGuIX NPs (producingAGuIX@PS@KDKPPR). After the synthesis and characterization of NPs, their photophysical properties as well as their affinity for NRP-1 recombinant protein, dark cytotoxicity, in vitro and in vivo vascular selectivity 
and PDT efficiency were evaluated. All the results were compared with the one obtained for a scramble NP (bearing the KRPKPD peptide).

\section{Materials and methods General}

All reactions involving porphyrin compounds were performed in the dark. Reactions were monitored by thin-layer chromatography (TLC) using aluminum-backed silica gel plates (MachereyNagel ALUGRAM ${ }^{\circledR}$ SIL > G/UV254), and ultraviolet light was used to detect TLC spots. Product purifications were performed using Silica Gel 60 (230-400 mesh) for column chromatography and Geduran $60 \mathrm{H}$ Silica Gel (63-200 mesh) for flash column chromatography. Reverse-phase high-performance liquid chromatography (HPLC) was performed on ProStar HPLC (Varian, Agilent Technologies, Santa Clara, CA, USA). Analytical HPLC was done with a Pursuit 5- $\mathrm{C}_{18}$ column (2.5 $\mu \mathrm{m}, 4.6 \times 150 \mathrm{~mm}$; Varian) and preparative HPLC with Pursuit $5-\mathrm{C}_{18}$ column $(5 \mu \mathrm{m}, 21.2 \times 150 \mathrm{~mm})$, both using a photodiode array detector (UV-visible detection; Varian) and a fluorescence detector (Varian).

\section{Chemicals}

Unless other stated, all chemicals were purchased as the highest purity commercially available and were used without further purifications. 5-(4-carboxyphenyl)-10,15,20-triphenylporphyrin (PS) was purchased from PorphyChem (Dijon, France). The Fmoc-Arg(Pbf)-Wang resin, the Fmoc-Asp(OtBu)Wang resin, 9-fluorenyl-methoxy-carbonyl (Fmoc)-amino acids and $\mathrm{N}, \mathrm{N}, \mathrm{N}^{\prime}, \mathrm{N}^{\prime}$-Tetramethyl-O-(1H-benzotriazol-1-yl) uronium hexafluorophosphate) (HBTU) were purchased from Iris Biotech $\mathrm{GmbH}$ (Marktredwitz, Germany). The $N$-hydroxysuccinimide, $N, N^{\prime}$-dicyclohexylcarbodiimide, $N$-methylmorpholine (NMM), $N$-methylpyrrolidinone (NMP), $N$ - $\varepsilon$-maleimidocaproic acid, trifluoroacetic acid (TFA) and triisopropylsilane (TIPS) were purchased from Alfa Aesar (Haverhill, MA, USA). Sodium hydroxide (NaOH; 99.99\%), hydrochloric acid $(\mathrm{HCl} ; 36.5 \%-38 \%)$, phosphate-buffered saline (PBS; tablet), dimethyl sulfoxide (DMSO; >99.5\%) and Fmoc-Lys-OH HCl were purchased from Sigma-Aldrich (St Louis, MO, USA), Traut's reagent (2-iminothiolane hydrochloride; $>97.0 \%$ ) was purchased from Alfa Aesar. Ellman's reagent, 5,5-dithio-bis-(2-nitrobenzoic acid) (>98\%), was purchased from Thermo Fisher Scientific (Waltham, MA, USA). AGuIX particles were purchased from NH TherAguix (Lyon, France). All products were used without further purification. Only Milli-Q water $(\rho>18 \mathrm{M} \Omega \cdot \mathrm{cm})$ was used for the aqueous solution preparation.

\section{Synthesis of PS-NHS: 5-(4-carboxyphenyl succinimide ester)-I0, I5,20-(triphenyl) porphyrin}

In the dark and under inert atmosphere, PS-COOH (1 eq) was dissolved in $\mathrm{CH}_{2} \mathrm{Cl}_{2}(20-50 \mathrm{~mL})$, and $N$-hydroxysuccinimide (3 eq) and $N, N^{\prime}$-dicyclohexylcarbodiimide (3 eq) were added. The mixture was stirred at $40^{\circ} \mathrm{C}$, for $4 \mathrm{~h}$, under argon. Purification of the crude material was performed using a silica gel column with dichloromethane (DCM). PS-NHS was obtained, after recrystallization in DCM/hexane, as a purple solid with a yield of $75 \%$.

\section{Synthesis of Fmoc-Lys(PS)-OH: 2-(9H- fluoren-9-ylmethoxycarbonylamino)- 6-[5-(4-carboxyphenyl)- I0, I 5,20- triphenylporphyrin]-hexanoic acid}

$N$-hydroxysuccinimide-activated porphyrin PS-NHS (152.4 $\mathrm{mg}, 0.20 \mathrm{mmol})$ and triethylamine $(0.21 \mathrm{mmol}$, $28.3 \mu \mathrm{L}$ ) in $10 \mathrm{~mL}$ of $\mathrm{CH}_{2} \mathrm{Cl}_{2}$ were added in the dark and under a nitrogen atmosphere to a solution of Fmoc-Lys-OH, $\mathrm{HCl}$ $(82.8 \mathrm{mg}, 0.20 \mathrm{mmol})$ in a minimum of dimethyl formamide (DMF). After being stirred at ambient temperature for $24 \mathrm{~h}$, the solvent was removed and the crude material was purified by column chromatography on silica gel using $\mathrm{EtOH} / \mathrm{CH}_{2} \mathrm{Cl}_{2}$, $5 / 95(\mathrm{v} / \mathrm{v})$, as the eluent. Solvent was removed in vacuo, and the residue recrystallized using $\mathrm{CHCl}_{3} / n$-hexane. The pure compound was isolated as a purple solid (166.5 mg, 82\%).

\section{Synthesis of $\mathrm{H}-\mathrm{K}(\mathrm{PS}) \mathrm{DK}$ PPR-OH}

The side chains of aspartic acid, lysine and arginine were respectively protected by 2,2,4,6,7-pentamethyldihydrobenzofuran-5-sulfonyl (Pbf), 5-tert-butyl ester (OtBu) and $N$-tert-butyloxy carbonyl (Boc) groups. $\mathrm{H}-\mathrm{D}(\mathrm{OtBu}) \mathrm{K}(\mathrm{Boc})$ $\mathrm{PPR}(\mathrm{Pbf})-$ Wang resin was synthesized on a multichannel peptide synthetizer (Intavis AG, Köln, Germany), according to a classical Fmoc/ $t$ Bu solid phase methodology, using a Fmoc-Arg(Pbf)-Wang resin on a $100 \mu$ mol scale. Double coupling was performed using a threefold excess of $N$-Fmocamino acid, activation reagents HBTU (3 eq), NMP (3 eq) and NMM (9 eq) in DMF. The coupling of Fmoc-Lys(PS)-OH ( 2 eq used) was the only one to be stirred for 5 days. Final Fmoc protection was removed using piperidine. The resin obtained was dried under vacuum and then cleaved using TFA/TIPS/water (92.5/5/2.5\%) for $2 \mathrm{~h}$. The acidic resin was

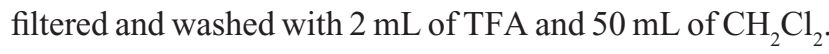
The filtrate was dried under vacuum and lyophilized. The crude product was further purified by preparative HPLC using 
acetonitrile/water $(0.1 \%$ TFA; $10: 90)$ to $100 \%$ acetonitrile gradient in $25 \mathrm{~min}$, followed by isocratic acetonitrile for $15 \min . R_{\mathrm{t}}=17.8 \mathrm{~min}$. Pure product was isolated as a red powder (15.7 mg, 11\%).

\section{Synthesis of the maleimido-PS@KDKPPR (maleimido-K(PS)DKPPR-OH)}

The side chains of aspartic acid, lysine and arginine were respectively protected by $\mathrm{Pbf}$, OtBu and Boc groups. $\mathrm{H}-\mathrm{D}(\mathrm{O} t \mathrm{Bu}) \mathrm{K}(\mathrm{Boc}) \mathrm{PPR}(\mathrm{Pbf})-\mathrm{Wang}$ resin was synthesized on a fully automated ResPepXL peptide synthesizer (Intavis AG), according to a classical Fmoc/tBu solid phase methodology, using a Fmoc-Arg(Pbf)-Wang resin on a $150 \mu \mathrm{mol}$ scale. Double coupling was performed using a threefold excess of $N$-Fmoc-amino acid, activation reagents HBTU (3 eq), NMP (3 eq) and NMM (9 eq) in DMF. After Fmoc deprotection by piperidine, the coupling of FmocLys(PS)-OH (1.5 eq used) was performed in presence of HBTU (3 eq), NMP (3 eq) and NMM (9 eq) in DMF, and the reaction mixture was stirred for 2 days. Final Fmoc protection was removed using piperidine, and the coupling of $N$ - $\varepsilon$-maleimidocaproic acid (1.2 eq) was realized in the same conditions as in the Fmoc-Lys(PS)-OH coupling. The resin obtained was dried under vacuum and then cleaved using TFA/TIPS/water (92.5/5/2.5\%) for $2 \mathrm{~h}$. The acidic resin was filtered and washed with $2 \mathrm{~mL}$ of TFA and $50 \mathrm{~mL}$ of $\mathrm{CH}_{2} \mathrm{Cl}_{2}$. The filtrate was dried under vacuum and lyophilized. The crude product was further purified by preparative HPLC using acetonitrile/water $(0.1 \%$ TFA; 10:90) to $100 \%$ acetonitrile gradient in $15 \mathrm{~min}$, followed by isocratic acetonitrile for $10 \mathrm{~min} . R_{\mathrm{t}}=17.8 \mathrm{~min}$. Pure product was isolated as a green powder (50 mg, $21 \%)$.

\section{AGuIX@SH synthesis and purification}

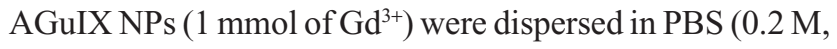
$\mathrm{pH} 8,10 \mathrm{~mL},\left[\mathrm{Gd}^{3+}\right]=100 \mathrm{mM}$ ) for $1 \mathrm{~h}$ at room temperature (RT). The Traut's reagent $(1 \mathrm{mmol})$ was dissolved in PBS (Traut's reagent $=50 \mathrm{mg} \cdot \mathrm{mL}^{-1}$ ) and added slowly to the NPs. The mixture was let under stirring at RT for $1 \mathrm{~h}$. Particles were then purified by tangential filtration trough Vivaspin ${ }^{\circledR}$ membranes (molecular weight cutoff $[\mathrm{MWCO}]=5 \mathrm{kDa}$ ) purchased from Sartorius Stedim Biotech (Aubagne, France). The colloidal solution was introduced into Vivaspin tubes and centrifuged; this step was repeated several times, by filling the tube and centrifuging. The purification process was followed by recording the absorbance spectra of the filtrated solution and stopped when no more signal at $248 \mathrm{~nm}$ was detected (signal due to the nonreacted Traut's reagent, total purification time $<1 \mathrm{~h}$ ). The $\mathrm{Gd}^{3+}$ yield was determined by inductively coupled plasma optical emission spectrometry (ICP-OES) and was of $80 \%$. The Traut yield was determined, thanks to the Ellman's reagent, and was of $8 \%$.

\section{Thiol quantification}

Several samples $(V=1 \mathrm{~mL})$ containing the same amount of NPs, $\mathrm{n}\left(\mathrm{Gd}^{3+}\right)=0.3 \mu \mathrm{mol}$, and an increasing quantity of Ellman's reagent (0-60 nmol) were prepared in PBS ( $\mathrm{pH} 8$ ). Samples were let in the dark for $15 \mathrm{~min}$ to react, and then their absorbance at $412 \mathrm{~nm}$ was measured using an UV-visible spectrophotometer (Varian Cary 50) and a Hellma semi micro cell $(1 \mathrm{~cm}$ light path, 1,400 $\mu \mathrm{L})$ manufactured from Suprasil quartz (Paris, France).

\section{AGulX@PS@KDKPPR synthesis and purification}

AGuIX@SH NPs $\left(500 \mu \mathrm{mol}\right.$ of $\left.\mathrm{Gd}^{3+}\right)$ were diluted in PBS ( $0.2 \mathrm{M}, \mathrm{pH} 8,6 \mathrm{~mL}$ ), and then, $1.4 \mathrm{~mL}$ of DMSO was added and the mixture heated at $40^{\circ} \mathrm{C}$. The maleimido-PS@ KDKPPR compound was dissolved in DMSO $(25 \mu \mathrm{mol}$, $15 \mathrm{mg} \cdot \mathrm{mL}^{-1}$ ) and added to the mixture in five additions, with a spaced out time of $30 \mathrm{~min}$. The additions were made drop by drop in the dark and under stirring. When all the maleimido-PS@KDKPPR compound was added, the solution was let under stirring for 2 more hours $\left(\left[\mathrm{Gd}^{3+}\right]=50 \mathrm{mM}\right)$. The solution was then diluted into water to reach a ratio of DMSO/water $<4 \%$ and purified through tangential filtration as previously described. The particles' solution was concentrated to approximately $\left[\mathrm{Gd}^{3+}\right]=100 \mathrm{mM}$, the $\mathrm{pH}$ was adjusted to 7.4 and, finally, the solution was sterilized by filtration through a $0.2 \mu \mathrm{m}$ syringe filter in order to remove the large impurities. It was freeze-dried for storage, using a Christ Alpha 1-2 lyophilizer (Osterode am Harz, Germany). The $\mathrm{Gd}^{3+}$ yield was determined by ICP-OES and was of $45 \%$. The maleimido-PS@KDKPPR yield was determined by absorbance measurements and was of $60 \%$. The same procedure was followed to synthesize AGuIX@PS@scramble.

\section{Affinity to NRP-I}

The surface of microplates (Corning Inc., Corning, NY, USA) was coated overnight at RT with NRP-1 at a concentration of $2 \mu \mathrm{g} / \mathrm{mL}$ (R\&D Systems, Inc., Minneapolis, MN, USA) in PBS. A solution of PBS containing $0.5 \%$ bovine serum albumin (BSA; blocking buffer) was then used to block the plates, to prevent nonspecific interactions, during $1 \mathrm{~h}$ at $37^{\circ} \mathrm{C}$. In all, $5 \mathrm{ng} / \mathrm{mL}$ of biotinylated VEGF-A 165 (R\&D Systems, Inc.) in blocking buffer containing $2 \mu \mathrm{g} / \mathrm{mL}$ of heparin was used to assess the binding of compounds to NRP-1. Biotinylated VEGF- $\mathrm{A}_{165}$ was added to the coated wells in competition with 
the different compounds or excess of non-labeled VEGF-A ${ }_{165}$, as a positive control. The plates were washed after a $2 \mathrm{~h}$ incubation at RT, and the amount of bound biotinylated VEGF-A ${ }_{165}$ was stained with streptavidin horseradish peroxidase conjugate (R\&D Systems, Inc.). The reaction was stopped after $20 \mathrm{~min}$ at RT by the addition of Stop Solution (R\&D Systems, Inc.). Optical densities were measured at $450 \mathrm{~nm}$. Results were expressed as relative absorbance to wells containing only blocking buffer. Three wells per condition were used.

\section{Cellular uptake of NPs}

Human umbilical vein endothelial cells (HUVEC) obtained from Merck Millipore (Billerica, MA, USA) were cultivated in the EndoGRO-LS complete medium (Merck Millipore) seeded in 24 -well plates at $5 \times 10^{4}$ cells/well. After 1 day, the medium containing NPs at the concentration of $1 \mu \mathrm{M}$ was added. After 4 or $24 \mathrm{~h}$, the medium was removed and cells were rinsed twice with cold PBS and trypsinized. After cell counting on a hemocytometer, cells were centrifuged $(1,200 \times g$ for $5 \mathrm{~min})$ and resuspended in lysis buffer $(0.2 \mathrm{~N} \mathrm{NaOH}, 0.5 \%$ Triton X-100). Centrifugation $(3,500 \times g$ for $15 \mathrm{~min})$ was used to remove cell debris. Fluorescence (recorded at $650 \mathrm{~nm}$ following excitation at $420 \mathrm{~nm}$ ) was measured on an M200 Pro spectrofluorometer (Tecan, Männedorf, Switzerland). Results are given as the fluorescence intensity by each cell. Experiments were carried out at least in triplicates.

\section{Dark cytotoxicity}

Cell survival after incubation with NPs in the dark was measured using a 3-(4,5-dimethylthiazol-2-yl)-2,5-diphenyl tetrazolium bromide (MTT) assay. Briefly, HUVEC were plated at an initial cell density of $5 \times 10^{4}$ cells/well in 24-well plates and allowed to attach overnight. Wells were rinsed twice with PBS and filled with $500 \mu \mathrm{L}$ of HUVEC medium containing various concentrations of NPs (from 0.1 to $10 \mu \mathrm{M}$ ). After a $24 \mathrm{~h}$ incubation at $37^{\circ} \mathrm{C}$, wells were rinsed twice with cold PBS and filled with $200 \mu \mathrm{L}$ of HUVEC medium. Cell survival was measured by MTT assay as previously described. ${ }^{29}$ Experiments were carried out in triplicates.

\section{Photocytotoxicity}

For photocytoxicity, NPs at a final concentration of 1 or $10 \mu \mathrm{M}$ were added in each well containing HUVEC previously seeded at $5 \times 10^{4}$ cells/well. HUVEC were cultivated for 4 or $24 \mathrm{~h}$ by keeping in darkness. Then, the cells were washed three times with the growth medium to remove the un-internalized NPs. After that, the cells were either exposed to various doses of light ( 5 or $10 \mathrm{~J} \cdot \mathrm{cm}^{-2}$ ) using a diode laser (Ceralas PDT 652; CeramOptec GmbH, Biolitec, Germany) to assess the photo-induced cytotoxicity of the NPs@PS. Irradiation was carried out at $652 \mathrm{~nm}$ with an irradiance of $4.54 \mathrm{~mW} / \mathrm{cm}^{2}$. Finally, cell survival was measured by MTT assay $24 \mathrm{~h}$ post irradiation as previously described. ${ }^{29}$ Each concentration of NPs was tested in sextuplicate.

\section{In vivo experiments: selectivity of NPs in a dorsal skinfold chamber}

All in vivo experiments were performed in accordance with the European Community guidelines (2010/63/EU) for the use of experimental animals with respect to the $3 \mathrm{Rs}$ ' requirements for Animal Welfare (the research project nos APAFIS 2368 and 2194 were approved by the French Ministry of Research). Animals were kept under standard conditions $\left(T=21^{\circ} \mathrm{C} \pm 1{ }^{\circ} \mathrm{C}\right.$, hygrometry $60 \% \pm 10 \%$, light on from 6 am to $6 \mathrm{pm}$ ) and had free access to standard diet and water.

Stereotactic U87 xenograft implantation was performed as previously described in Toussaint et al. ${ }^{25} \mathrm{MRI}$ acquisitions were performed using a small animal 7 Tesla magnet (BioSpec 70/20 USR; Bruker Optik GmbH, Ettlingen, Germany). The most common MRI sequences and T1-weighted imaging (RARE acquisition; relaxation enhancement $\mathrm{TR} / \mathrm{TE}=400 / 9 \mathrm{~ms}$; matrix: $256 \times 256$ ) were repeated in axial planes before injection and also at different times after AGuIX@PS@KDKPPR intravenous injection (4 $\mu \mathrm{mol} \cdot \mathrm{kg}^{-1}$, porphyrin equivalent) to visualize the tumor tissue in positive signal. For selectivity of NPs using the dorsal skinfold chamber model, 8-10 weeks old nude female mice were used (Janvier, Le Genest-Saint-Isle, France). U87 xenografts were obtained and implanted in skinfold chambers as previously described in Tylcz et al. ${ }^{35}$ After 10-14 days following U87 cells implantation, mice were divided into three batches: a batch receiving AGuIX@PS@KDKPPR NPs, another one with AGuIX@PS@scramble and the last one with AGuIX@ PS NPs. Visualization of the localization of different NPs into the vasculature (lumen versus vascular column) was performed on anesthetized mice bearing skinfold chambers at 1, 6 and $24 \mathrm{~h}$ after injection using the Nikon microscope AZ100 (Nikon Corp., Tokyo, Japan) system equipped with a Digital Light DS-Qi1Mc camera and a confocal device (Revolution DSD; Andor Technology, Belfast, UK).

\section{Statistical analysis}

All results were given as mean \pm standard error of the mean (SEM). Nonparametric Mann-Whitney $U$ test was used to determine the statistical significance with a limit set to $P<0.05$ using GraphPad Prism 5 (GraphPad Software, Inc., La Jolla, CA, USA) versus AGuIX@PS@scramble. 


\section{Results}

The syntheses of AGuIX and AGuIX functionalized with 5-(4-carboxyphenyl)-10,15,20-triphenylporphyrin (AGuIX@PS) NPs have already been described in the literature. ${ }^{25,36}$ In the present study and in order to maintain the affinity of the DKPPR peptide for NRP-1 recombinant protein, a new functionalization approach has been suggested. It consisted of the conjugation of the KDKPPR peptide moiety to the PS molecules, followed by the grafting of this new PS@peptide conjugate to the surface of the AGuIX NPs through a maleimido-thiol reaction. It producedAGuIX@PS@KDKPPR NP. The same protocol was also used with a scramble peptide containing the same amino acids (maleimido-PS@KRPKPD, maleimido-PS@ scramble) to check the targeting selectivity of this new nanoplatform.

\section{Synthesis and characterization of peptide derivatives}

Maleimido-PS@KDKPPR and maleimido-PS@scramble compounds were synthesized by Fmoc/tBu solid-phase strategy (Figure 1). It is known that the lysine side chain provides a convenient handle to link a PS. For the synthesis of the maleimido-PS@KDKPPR and maleimido-PS@scramble,we choose to link first in liquid phase the 5-(4-carbonylphenyl)10,15,20-triphenyl-porphyrin succidinyl ester (PS-NHS) already described by our team ${ }^{37}$ to the amine function of the lysine lateral side chain to form the Fmoc-Lys(PS)-OH (Figure 1). The coupling of Fmoc-Lys(PS)-OH and $N-\varepsilon-$ maleimidocaproic acid was achieved on a H-peptide-Wang resin. During the coupling of Fmoc-Lys(PS)-OH and all the next steps, light exposure was minimized to limit unwanted side reactions by sealing the vessel in aluminum foil. The maleimido-PS@KDKPPR and maleimido-PS@scramble peptides were cleaved from the resin with a mixture of TFA, TIPS and water(92.5/2.5/5). The maleimido-PS@KDKPPR and maleimido-PS@scramble compounds were purified by preparative reversed-phase (RP)-HPLC. Characterizations of the compounds were confirmed by high-resolution mass spectrometry (HRMS) and ${ }^{1} \mathrm{H}$ NMR experiments (Supplementary $\underline{\text { materials }}$ ). As shown in Figure 2A, the photophysical properties of PS are preserved during the maleimido-PS@KDKPPR compound synthesis; indeed, the singlet oxygen and the fluorescence quantum yields remain almost identical. The

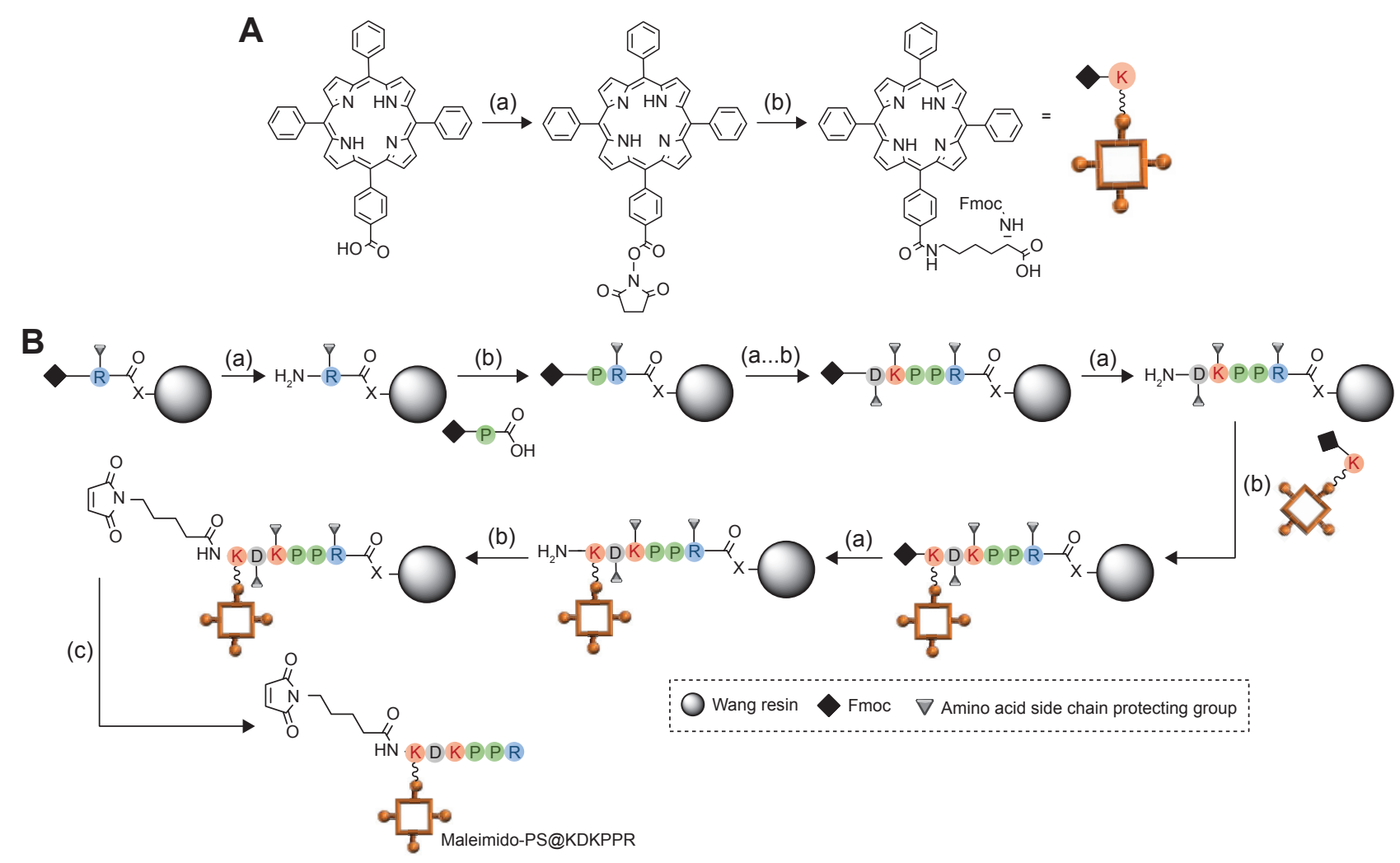

Figure I PS synthesis.

Notes: (A) Preparation of Fmoc-Lys(PS)-OH: (a) DCC, NHS, $\mathrm{CH}_{2} \mathrm{Cl}_{2}, 40^{\circ} \mathrm{C}, 4 \mathrm{~h}$ and (b) Fmoc-Lys-OH HCl, Et ${ }_{3} \mathrm{~N}, \mathrm{DCM}, \mathrm{DMF}, 0^{\circ} \mathrm{C}$ to RT, 24 h. (B) Solid phase synthesis of the PS@KDKPPR compound: (a) N-deprotection: 20\% piperidine in DMF, (b) coupling: HBTU/NMM/NMP and (c) cleaving: TFA/TIPS/ $\mathrm{H}_{2} \mathrm{O}$.

Abbreviations: PS, photosensitizer; DCM, dichloromethane; RT, room temperature; NMM, $N$-methylmorpholine; NMP, N-methylpyrrolidinone; TFA, trifluoroacetic acid; TIPS, triisopropylsilane; DCC, N,N'-dicyclohexylcarbodiimide; DMF, dimethyl formamide; NHS, N-Hydroxysuccinimide; HBTU, N,N,N',N'-Tetramethyl-O-(I H-benzotriazolI-yl)uronium hexafluorophosphate. 
A

\begin{tabular}{|c|c|c|c|c|c|}
\hline & & PS & PS@KDKPPR & $\begin{array}{l}\text { Maleimido-PS } \\
\text { @KDKPPR }\end{array}$ & $\begin{array}{l}\text { Maleimido-PS } \\
\text { @scramble }\end{array}$ \\
\hline \multirow{5}{*}{$\begin{array}{l}\varepsilon\left(10^{3}\right) \\
\left(\mathrm{L} \cdot \mathrm{mol}^{-1} \cdot \mathrm{cm}^{-1}\right)\end{array}$} & $\begin{array}{l}\text { Soret } \\
\text { band }\end{array}$ & 224 & 341 & 331 & 210 \\
\hline & $\mathbf{Q}_{\mathrm{IV}}$ & 15.1 & 13.4 & 11.9 & 9.6 \\
\hline & $\mathbf{Q}_{\mathrm{III}}$ & 7.76 & 5.93 & 5.03 & 4.42 \\
\hline & $\mathbf{Q}_{\|}$ & 5.31 & 3.72 & 2.83 & 2.87 \\
\hline & $\mathbf{Q}_{1}$ & 4.49 & 3.17 & 2.2 & 2.5 \\
\hline$\phi_{\mathrm{F}}(\%)$ & & 15 & 13 & 13 & 13 \\
\hline$\phi_{\Delta}(\%)$ & & 24 & 21 & 20 & 19 \\
\hline
\end{tabular}

B

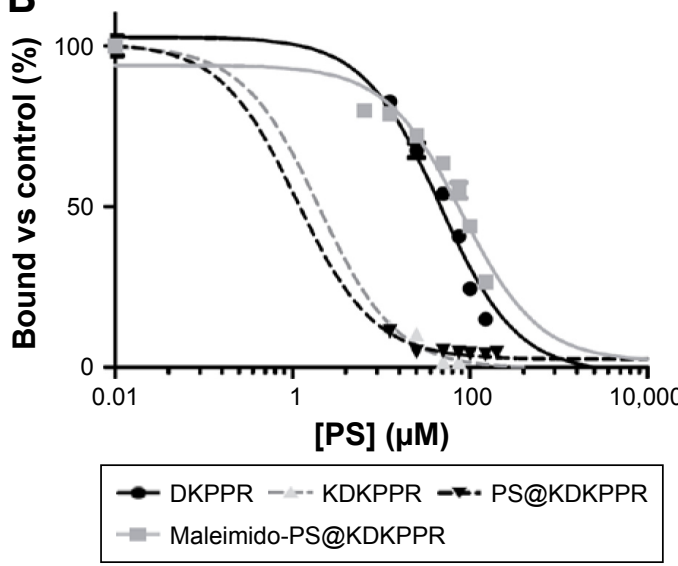

Figure 2 PS characterizations.

Notes: (A) Absorption coefficient, fluorescence and singlet oxygen quantum yields of PS, PS@KDKPPR, Maleimido-PS@KDKPPR, Maleimido-PS@scramble. (B) Binding of the DKPPR peptide, the KDKPPR peptide, the PS@KDKPPR and the maleimido-PS@KDKPPR to recombinant NRP-I protein. Binding of biotinylated VEGF (5 ng/mL; $110 \mathrm{pM})$ to NRP-I in the presence of $2 \mu \mathrm{g} / \mathrm{mL}$ heparin was evaluated when increasing concentrations of compounds (6.5-200 $\mu \mathrm{M})$ were added (data points show the mean \pm SD, $\mathrm{n}=3$ ). The values found for $\mathrm{EC}_{50}$ are $46 \mu \mathrm{M}$ for DKPPR, $2 \mu \mathrm{M}$ for KDKPPR, I $\mu \mathrm{M}$ for PS@KDKPPR and 8I $\mu \mathrm{M}$ for maleimido-PS@KDKPPR.

Abbreviations: PS, photosensitizer; DMSO, dimethyl sulfoxide; $\mathrm{EC}_{50}$, concentration of competitor that displaced $50 \%$ of biotinylated VEGF- $\mathrm{A}_{165}$ binding.

affinity of the peptide for the NRP-1 receptor was estimated as $\mathrm{EC}_{50}$ values (ie, the concentration of competitor that displaced $50 \%$ of biotinylated VEGF-A ${ }_{165}$ binding, using the medium effect method). It was found to be almost as good as one of KDKPPR alone (Figure 2B). Indeed, we found an $\mathrm{EC}_{50}$ value of $2 \mu \mathrm{M}$ for KDKPPR, $1 \mu \mathrm{M}$ for PS@KDKPPR and $81 \mu \mathrm{M}$ for maleimido-PS@KDKPPR.

\section{Synthesis and characterization of the NPs for VTP}

To introduce thiol group on the NP, a cyclic imido ester, the Traut's reagent, was incubated with AGuIX NPs for $1 \mathrm{~h}$ at $\mathrm{RT}$ and at $\mathrm{pH} 8$ (Figure $3 \mathrm{~A}$ ). It reacted with the primary amines present at the surface of the particles in a ring-opening reaction, resulting in a free sulfhydryl..$^{38}$ The obtained AGuIX@SH particles were then purified by tangential filtration over a $5 \mathrm{kDa}$ cutoff membrane. Thanks to the absorbance of Traut's reagent at $248 \mathrm{~nm}$, the purification was followed by UV-visible spectroscopy. In order to quantify the number of thiols per particle, the Ellman's reagent was used..$^{39}$ In slightly basic conditions, it can react with sulfhydryl groups and release the 5-thio-2-nitrobenzoic acid, presenting a strong absorbance at $412 \mathrm{~nm}$ (Figure 3B). Briefly, the quantification is based on the addition of an increasing quantity of Ellman's reagent to AGuIX@SH particles. First, an increase in absorbance at $412 \mathrm{~nm}$ can be observed because of the reaction of thiol with Ellman's reagent. When all the thiol functions have reacted, a plateau is reached. The results give approximately one thiol for $10 \mathrm{Gd}^{3+}$ (corresponding to about one per particle).
In the second step, as shown in Figure 4A, AGuIX@ SH was functionalized with the maleimido-PS@KDKPPR compound by creating a stable thioester bond. To obtain solubility of both NPs and porphyrin derivatives, the maleimido-PS@KDKPPR was gradually added to the NPs in a mixture of PBS and DMSO at $40^{\circ} \mathrm{C}$. Then, the colloidal suspension was purified by tangential filtration over a $5 \mathrm{kDa}$ cutoff membrane and by filtration over a $0.2 \mu \mathrm{m}$ filter. As presented in Figure $4 \mathrm{~B}$, the resulting AGuIX@PS@KDKPPR NPs have hydrodynamic diameter of $\sim 10 \mathrm{~nm}$, which is suitable for renal elimination. Besides, magnetic properties are consistent with their use as a positive contrast agent in MRI $\left(r_{1}=16.1 \mathrm{~s}^{-1} \cdot \mathrm{mM}^{-1}\right.$ per $\mathrm{Gd}^{3+}, r_{2} / r_{1}=1.6$ at $37^{\circ} \mathrm{C}$ and $\left.1.4 \mathrm{~T}\right)$. No modification in the maleimido-PS@KDKPPR absorbance spectra was detected after grafting (Supplementary materials). Calibration curves indicate that one maleimido-PS@KDKPPR compound is present for $15 \mathrm{Gd}^{3+}$ (corresponding to one to two particles). However, the fluorescence spectrum was found to be modified (refer Supplementary materials for the emission spectra after an excitation at $420 \mathrm{~nm}$ ). Because of the poor solubility of PS in water, its fluorescence $\left(\Phi_{\mathrm{F}}\right)$ and singlet oxygen $\left(\Phi_{\Delta}\right)$ quantum yield as well as the one for maleimido-PS@KDKPPR compound cannot be determined in water. As presented in Figure $4 \mathrm{~B}$, this is no more the case when maleimido-PS@KDKPPR is grafted on AGuIX ( $\Phi_{\mathrm{F}}=10 \%$ and $\Phi_{\Delta}=28 \%$ ), highlighting the fact that AGuIX enables the solubility of PS in water. Besides, these yields are similar to the only PS or maleimido-PS@KDKPPR in $\operatorname{DMSO}\left(\Phi_{\mathrm{F}}=15\right.$ and $13 \%$ or $\Phi_{\Delta}=24$ and $20 \%$ respectively). 
A

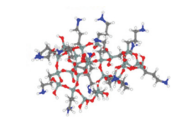

Polysiloxane
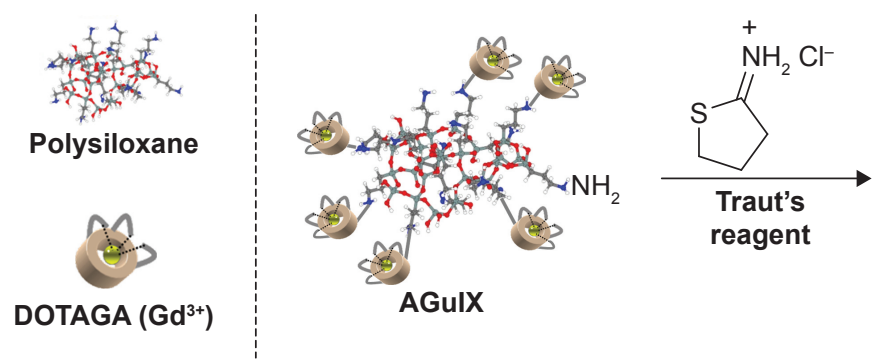

$\mathrm{NH}_{2} \mathrm{Cl}^{-}$

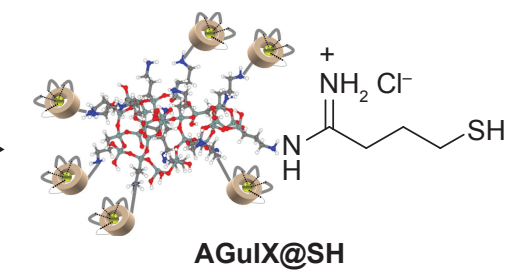

B
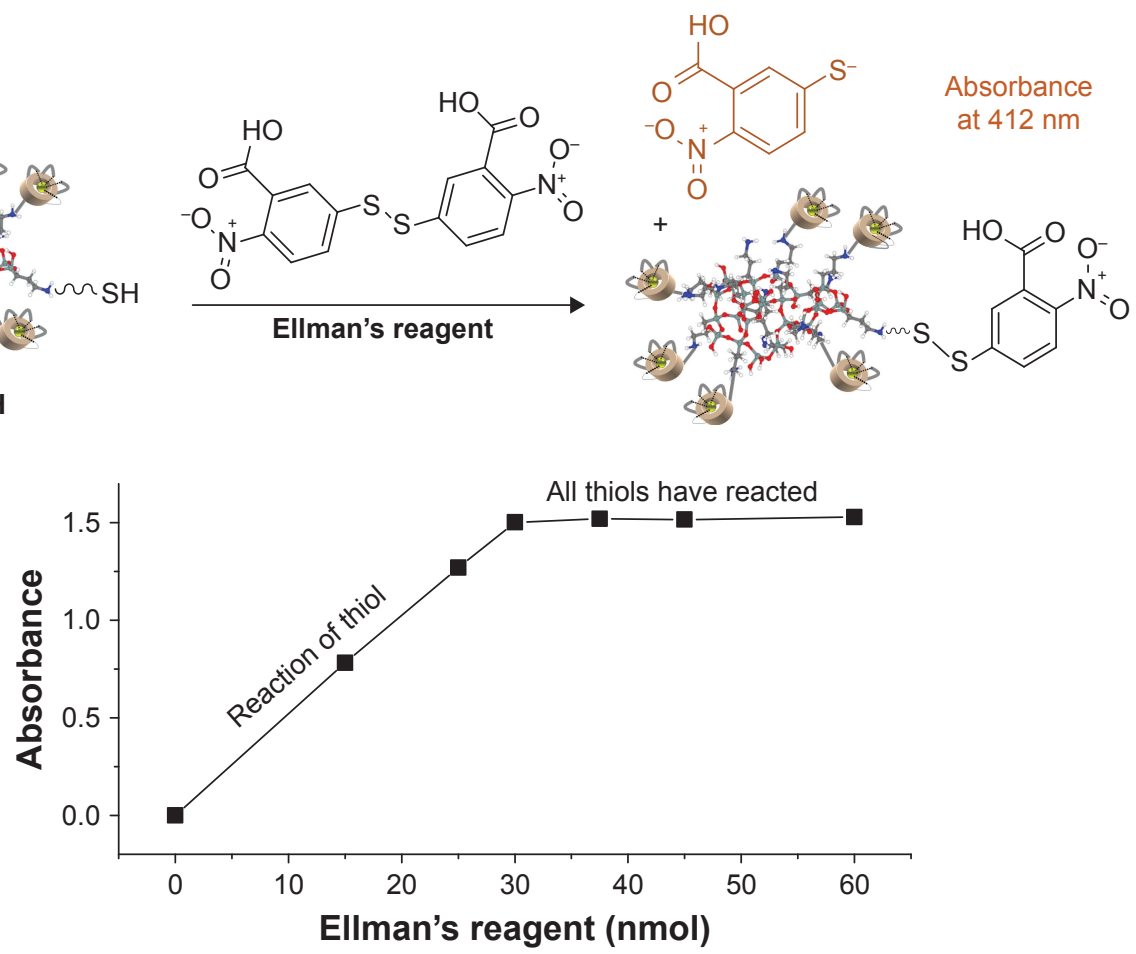

Figure 3 AGulX@SH NPs synthesis and characterizations.

Notes: (A) Reaction of the amino group with the Traut's reagent (PBS, pH =8, [Gd $\left.{ }^{3+}\right]=80 \mathrm{mM}$, RT, one Traut's reagent for one Gd). (B) Thiol quantification on AGulX@SH using the Ellman's reagent and absorbance measurements, $\mathrm{n}\left(\mathrm{Gd}^{3+}\right)=0.3 \mu \mathrm{mol}$ in PBS $(\mathrm{pH}=8), 15 \mathrm{~min}, \mathrm{RT}$. The change of slope indicates that there are $\sim 30 \mathrm{nmol}$ of thiol in the solution (one SH for one particle).

Abbreviations: NP, nanoparticle; PBS, phosphate-buffered saline; RT, room temperature.

The NPs conjugated with the scramble peptide, AGuIX@ PS@scramble (AGuIX@PS@KRPKPD), present similar characteristics.

After intravenous injection of KDKPPR-targeted NPs, the positive contrast enhancement by MRI allowed us to visualize the tumor tissue (Figure 4C). Owing to these multifunctional NPs, we optimized the position of the optical fiber into the tumor tissue and selected an optimal drug-light interval between the nanoplatforms injection and the photodynamic treatment, validating the concept of iPDT guided in real time by MRI analysis.

\section{NPs affinity to NRP-I protein}

For measurements of the binding affinities of NPs for recombinant NRP-1 protein, a competitive binding test using biotinylated VEGF-A ${ }_{165}$ was performed. As biotinylated VEGF- $\mathrm{A}_{165}$ binding to this receptor is heparin dependent, the competitive binding experiments were always carried out in the presence of heparin. As shown in Figure 5,AGuIX@PS@ KDKPPR displaces the binding of biotinylated VEGF- $\mathrm{A}_{165}$ to NRP-1 $\left(\mathrm{EC}_{50}=19 \mu \mathrm{M}\right)$ more effectively than maleimido-PS@ KDKPPR and AGuIX@PS@scramble (81 and $176 \mu \mathrm{M}$, respectively).

\section{In vitro dark cytotoxicity}

An MTT test was used to evaluate the dark cytotoxicity of NPs for PS concentration, ranging from 1 to $10 \mu \mathrm{M}$ in HUVEC. In the absence of light exposure and after $24 \mathrm{~h}$ incubation with $10 \mu \mathrm{M}$ of NPs@PS, NPs exhibited no cytotoxicity (Supplementary materials). 
A

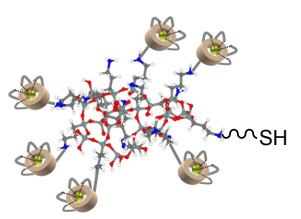

$\sim 1 \mathrm{SH}$ for $10 \mathrm{Gd}^{3+}$

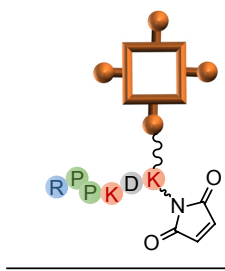

Maleimido-PS@ KDKPPR

AGuIX@SH

B

\begin{tabular}{ll}
\hline & AGulX \\
\hline $\begin{array}{l}\text { Hydrodynamic } \\
\text { diameter }\end{array}$ & $D_{\mathrm{H}}=3.3 \pm 0.8 \mathrm{~nm}$ \\
Relaxometry & $r_{1}=12.4 \mathrm{~s}^{-1} \cdot \mathrm{mM}^{-1}$ \\
$\left(60 \mathrm{mHz} 37^{\circ} \mathrm{C}\right)$ & $r_{2} / r_{1}=1.4$ \\
$\phi_{\mathrm{F}}(\%)$ & - \\
$\phi_{\Delta}(\%)$ & -
\end{tabular}

C

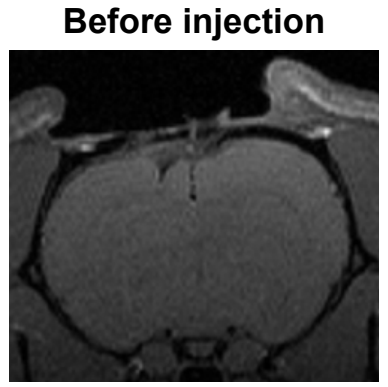

$1 \mathrm{~h}$ after injection

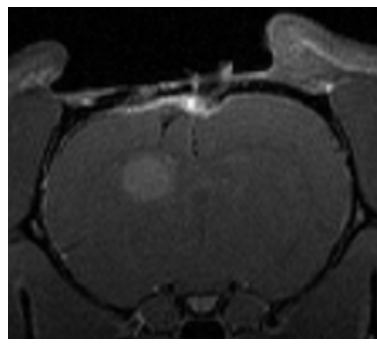

$6 \mathrm{~h}$ after injection

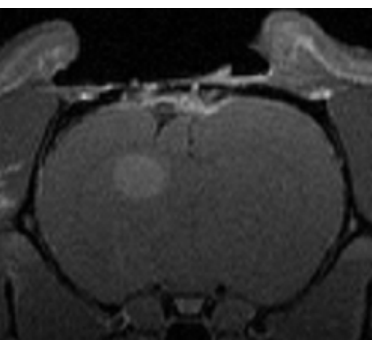

$24 \mathrm{~h}$ after injection

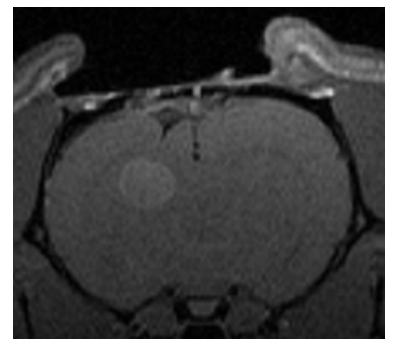

Figure 4 Synthesis and characterizations of AGulX@PS@KDKPPR NPs.

Notes: (A) Grafting of the maleimido-PS@KDKPPR compound on AGulX@SH NPs, DMSO/PBS (pH=8), $40^{\circ} \mathrm{C}, 4$ h, [G ${ }^{3+}$ ] =50 mM, one PS for two SHs. After purification, approximately one PS molecule has been conjugated to one to two NPs. For AGulX@PS@scramble NPs, the same procedure was followed and similar results were obtained. (B) Size and magnetic properties of AGulX, AGulX@SH, AGulX@PS@KDKPPR and AGulX@PS@KDKPPR NPs. All NPs described a mean hydrodynamic diameter < or $\sim 10 \mathrm{~nm}$ and magnetic properties consistent with their real interest as a positive contrast agent for MRI analysis. AGulX@PS@KDKPPR and AGulX@PS@ scramble NPs describe similar fluorescence quantum yields $\left(\Phi_{\mathrm{F}}\right)$ and singlet oxygen quantum yields $\left(\Phi_{\Delta}\right)$ in $\mathrm{D}_{2} \mathrm{O}$. (C) TI coronal MRI images obtained before AGulX@PS@ KDKPPR NPs intravenous injection and I, 6 and $24 \mathrm{~h}$ after intravenous injection. Proton density weighted MRI (TR/TE: 5000/33 ms, NEX: 2, FOV: 4×4 cm, matrix: 256×256, SI: I mm). Maximal MRI signal intensity after injection was observed I h post injection.

Abbreviations: PS, photosensitizer; NP, nanoparticle; DMSO, dimethyl sulfoxide; PBS, phosphate-buffered saline; MRI, magnetic resonance imaging.

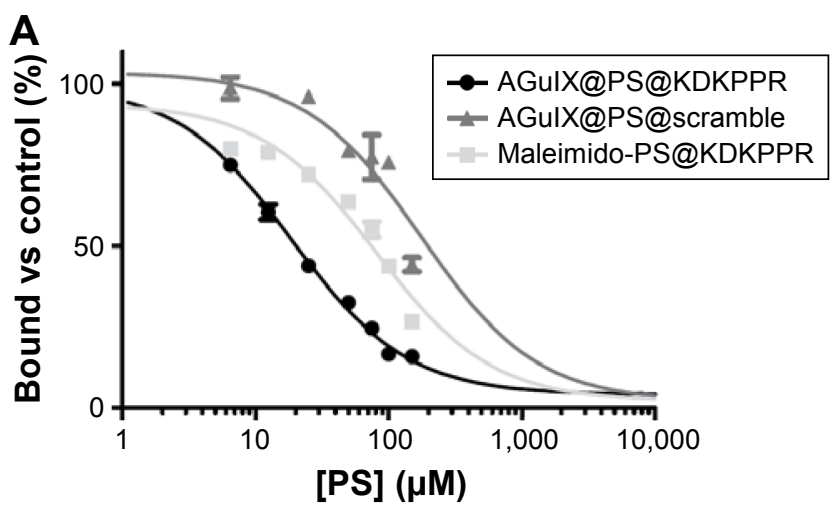

B

\begin{tabular}{ll}
\hline Compounds & $\mathrm{EC}_{50}(\boldsymbol{\mu M})$ \\
\hline Maleimido-PS@KDKPPR & 81 \\
AGuIX@PS@KDKPPR & 19 \\
AGulX@PS@scramble & 176 \\
\hline
\end{tabular}

Figure 5 Binding of AGulX@PS, AGulX@PS@scramble and AGulX@PS@KDKPPR NPs to recombinant NRP-I protein.

Notes: (A) Binding of biotinylated VEGF $165(5 \mathrm{ng} / \mathrm{mL}$; $110 \mathrm{pM})$ to NRP-I in the presence of $2 \mu \mathrm{g} / \mathrm{mL}$ heparin was evaluated when increasing concentrations of peptide (6.5-200 $\mu$ M) were added (data points show the mean \pm SD, $n=3$ ). (B) AGulX@PS@KDKPPR NPs lead to a relevant affinity toward NRP-I protein compared to maleimidoPS@KDKPPR or AGulX@PS@scramble.

Abbreviations: PS, photosensitizer; NP, nanoparticle; $\mathrm{EC}_{50}$, concentration of competitor that displaced $50 \%$ of biotinylated VEGF-A ${ }_{165}$ binding. 


\section{In vitro and in vivo selectivity}

The cellular uptake of NPs was examined using HUVEC, in the dark, at a PS non-cytotoxic concentration of $1 \mu \mathrm{M}$. As shown in Figure 6A, after $4 \mathrm{~h}$ of NPs' incubation with HUVEC cells, the AGuIX@PS@KDKPPR uptake was found to be twice higher than AGuIX@PS or AGuIX@PS@scramble NPs in relation with the grafting of DKPPR peptide. Indeed, the fluorescence per cell is $7.85 \pm 1.89 \times 10^{-3}, 3.83 \pm 0.75 \times 10^{-3}$ and $3.25 \pm 0.42 \times 10^{-3}$, respectively. However, no statistically relevant difference in cellular uptake was observed after $24 \mathrm{~h}$ of incubation for AGuIX@PS and AGuIX@PS@KDKPPR (fluorescence per cell of $2.52 \pm 0.55 \times 10^{-2}$ and $2.58 \pm 0.29 \times 10^{-2}$, respectively).

The vascular distribution of the NPs was performed by intravital microscopy in U87 xenograft tumors implanted in nude mice skinfold chambers. In vivo, distribution of
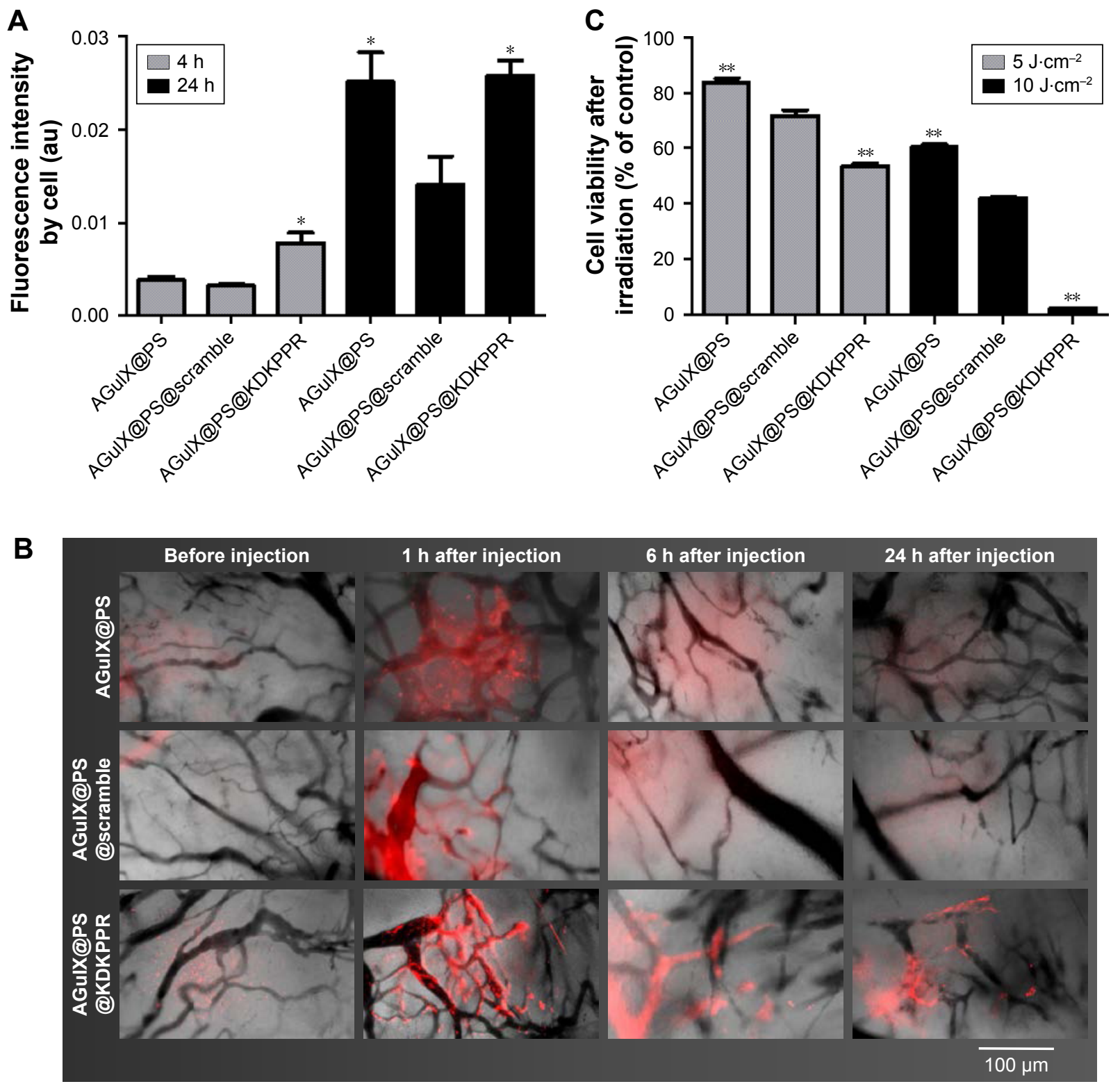

Figure 6 In vitro and in vivo selectivity followed by the photodynamic efficiency of functionalized NPs on HUVEC.

Notes: (A) In vitro selectivity: uptake in HUVEC after 4 and $24 \mathrm{~h}$ of incubation with functionalized NPs ([PS] $=I \mu M)$. The fluorescence measured before injection is related to the tissue autofluorescence (measured before administration of NPs). (B) In vivo selectivity: selectivity of NPs using a dorsal skinfold chamber model, before and I, 6 and 24 h after intravenous injection of AGulX@PS, AGulX@PS@scramble and AGulX@PS@KDKPPR ([PS]=6 $\mu$ mol/kg). Blood vessels are represented in black and PS fluorescence in red. On the contrary to AGulX@PS or AGulX@PS@scramble, thanks to the targeting peptide, AGulX@PS@KDKPPR NPs were fixed to the vessel walls of the tumor tissue. All pictures have been taken with the same magnification, scale bar represents $100 \mu \mathrm{m}$. (C) Influence of light dose on cell viability after PDT (5 or $10 \mathrm{~J} \cdot \mathrm{cm}^{-2}$ ), following exposure of NPs ([PS] =I $\mu$ M and exposition time of $4 \mathrm{~h}$ ). For in vitro experiments, $\mathrm{n}=3$ or $\mathrm{n}=6$. *P $<0.05$ and $* * P<0.01$ versus AGulX@PS@scramble. A statistically significant photocytotoxicity was obtained with AGulX@PS@KDKPPR.

Abbreviations: NP, nanoparticle; HUVEC, human umbilical vein endothelial cells; PS, photosensitizer; au, atomic unit. 
AGuIX@PS and AGuIX@PS@scramble describes the same profile (Figure 6B). They were found free in the blood vessels at $1 \mathrm{~h}$ but disappeared from them at 6 h. AGuIX@PS@ KDKPPR NPs were recognized by the tumor endothelium and seem to be bound to the vessels walls of the tumor from $1 \mathrm{~h}$ postintravenous injection. The binding remains visible by fluorescence until $24 \mathrm{~h}$. These results look very promising in the context of VTP.

\section{In vitro PDT efficiency}

Figure 6C illustrates in vitro results concerning photocytotoxicity on HUVEC and light dose efficiency. As expected, increasing light dose leads to a decrease in cell viability. Indeed, the cell viability ( $\%$ ) for an irradiation at $5 \mathrm{~J} \cdot \mathrm{cm}^{-2}$ was $100 \pm 6,84 \pm 3,72 \pm 5$ and 53 \pm 3 for the control, AGuIX@PS, AGuIX@PS@scramble and AGuIX@PS@KDKPPR NPs, respectively. At $10 \mathrm{~J} \cdot \mathrm{cm}^{-2}$, it was $100 \pm 3,60 \pm 2,42 \pm 1$ and $2 \pm 1$ for the control,AGuIX@PS,AGuIX@PS@scramble and AGuIX@PS@KDKPPR, respectively. The best results were obtained with AGuIX@PS@KDKPPR compared to other NPs, illustrating the real interest of the DKPPR-targeting peptide for PDT. The effect of incubation time or concentration (of porphyrin) was also studied (Supplementary materials). As expected by the results of HUVEC cellular uptake, it was found that after $4 \mathrm{~h}$ of incubation time, a relevant photocytotoxic effect was obtained with AGuIX@PS@KDKPPR compared with AGuIX or AGuIX@PS.

\section{Discussion}

Many studies have shown that tumor vasculature targeting is effective in enhancing the therapeutic efficacy of PDT. Several strategies exist as the use of antibodies or small synthetic peptides have the advantages of being easier to prepare and handle. ${ }^{40,41}$ For example, Ichikawa et al ${ }^{42}$ have synthesized liposomes bearing a pentapeptide (APRPG) and a PS (benzoporphyrin derivative) for the targeting of angiogenic endothelial cells. They showed that peptideconjugated liposomal PS exhibited strong suppression of tumor growth compared to those targeted with NPs without APRPG. Besides, there is a growing interest in the development of particles for VTP and imaging. Kopelman et $\mathrm{al}^{43}$ were the first to describe the synthesis of a nonbiodegradable NP for the diagnosis and treatment of brain cancer. These nanosystems were composed of polyacrylamide, MRI contrast agents (for imaging), PSs (for PDT), PEG chains (for a better solubility and biodistribution) and an RGD peptide moiety (for the targeting of neovasculature). In vivo experiments on rats bearing intracerebral 9L tumors showed that the MRI contrast agent provided real-time tumor kill measurement.

The goal of our study was to design and to evaluate a new nanoplatform consisting of a surface-localized tumor vasculature targeting NRP-1 and displaying photodynamic and imaging agents for iPDT of GBM guided by interventional MRI. An NP called AGuIX was used; it is composed of gadolinium chelates, DOTAGA, 2,2',2"'-(10-(2,6-dioxotetrahydro2H-pyran-3-yl)-1,4,7,10-tetraazacyclododecane-1,4,7-triyl) triacetic acid, covalently grafted to a polysiloxane core; its synthesis and properties have already been presented in the literature. ${ }^{36,44-48}$ A PS, a tetraphenylporphyrin (TPP) and a peptide (KDKPPR) targeting the NRP-1 receptor overexpressed by tumor angiogenic vessels were then grafted on the surface of this particle. The choice of the AGuIX NPs was driven by several points. First, they display a hydrodynamic diameter $<10 \mathrm{~nm}$, even after functionalization, which is small enough to provide exclusive renal excretion and well adapted for further clinical development. They can also accumulate in the tumor by the EPR effect. So far, no toxicity has been noticed during regulatory toxicity tests on rodents and monkeys, and AGuIX has been recently accepted for a Phase I clinical assay (EudraCT no: 2015-004259-30). The presence of gadolinium enables the NPs to be used as a positive MRI contrast agent; it will therefore be possible to detect the tumor tissue, to determine an accurate position for the PDT fiber and to follow up by noninvasive imaging photodynamic efficiency.

In this study, it was decided not to graft separately the PS and the targeting peptide on the particle but to graft directly the compound PS@KDKPPR. The major interest is to improve the synthesis reproducibility by performing only one conjugation step on the particle, to conserve a good homogeneity for the functionalized particles and, finally, to quantify easily the number of targeting peptides on the particle, thanks to the strong absorbance of the PS. AGuIX particles present primary amines on their surface, and our group has recently published a study in which these groups were used to graft a TPP molecule on AGuIX. ${ }^{25}$ In that case, the porphyrin used presented an NHS ester function that could react with an amino group to form a covalent amide bond. In the present study, a new functionalization approach had to be developed in order to maintain the affinity of the KDKPPR peptide for NRP-1 receptor. First, a thiol function was added to the surface of the particles and reacted in a second step with the maleimido-PS@KDKPPR compound to form a stable thioether bond. Maleimide reactions are specific for thiols at the used $\mathrm{pH}$ of $7.5 .{ }^{38}$ With this strategy, 
we can easily modulate the number of PS@KDKPPR per particle; in our case, one compound was added for $15 \mathrm{Gd}^{3+}$ (corresponding to approximately one PS for one or two NPs). With this ratio, a good solubility and colloidal stability in aqueous solutions were maintained and an appropriate PDT efficiency was obtained. Besides, the magnetic properties of the functionalized particles $\left(r_{2} / r_{1}=1.6 \mathrm{~s}^{-1} \cdot \mathrm{mM}^{-1}\right.$ at $37^{\circ} \mathrm{C}$ and $60 \mathrm{MHz}$ ) are consistent with their use as a positive contrast agent in MRI to guide and follow PDT treatment. The same process was used for the coupling of a scramble peptide (KRPKPD) coupled to the PS.

In vitro, in the absence of light irradiation, the functionalized NPs showed no cytotoxicity for the concentration of PS $<10 \mu \mathrm{M}$ using HUVEC overexpressing NRP-1 receptor. The singlet oxygen quantum yield was not altered by the grafting on the NPs, showing that the PS molecules could be photoactivated to yield photocytotoxic effects in vitro and in vivo ( $\Phi_{\Delta}=20 \%$ for maleimido-PS $@$ KDKPPR in DMSO and $\Phi_{\Delta}=28 \%$ for AGuIX@PS@KDKPPR in water). This is consistent with previous studies on this kind of NPs bearing chlorin or porphyrin PSs. ${ }^{11,25,29}$ The way by which the PS is added in or on the NP is really crucial because it can have an impact on its photophysical properties and therefore on its photocytotoxic effect. Chu et al designed silica-based NPs encapsulating methylene blue. They showed that the singlet oxygen formation with the particle was lower than with the methylene blue alone probably because of dimer's formation inside the particle. ${ }^{49}$ A comparative study was also performed by Tang et $a l^{50}$ to evaluate the encapsulation of methylene blue in two types of silica-based NPs. One was prepared using tetraethoxysilane and the other one using methyltrimethoxysilane and phenyltrimethoxysilane as precursors. If the PS loading was higher with the Stöber process, the kinetic of singlet oxygen formation was lower. Our strategy describes different advantages for the PS photophysical properties. As the PS is grafted on the surface of the particle, the singlet oxygen does not need to diffuse in and out of the particles and the grafting prevents the PS aggregation. The photocytotoxic effect was confirmed in vitro on HUVEC. Indeed, we observed that the presence of the peptide units (KDKPPR) onto the AGuIX@PS increased its PDT effect, probably due to an improved cellular uptake.

Molecular affinity was investigated for functionalized particles. Even after grafting on NPs, the KDKPPR peptide was still able to bind NRP-1 protein. Indeed, AGuIX@ PS@KDKPPR showed a relevant molecular affinity of $19 \mu \mathrm{M}$, whereas the maleimido-PS@KDKPPR demonstrated a molecular recognition for NRP-1 protein of $81 \mu \mathrm{M}$ (four times higher) and the scramble particles, AGuIX@PS@ scramble, had one of $176 \mu \mathrm{M}$ ( $\sim 10$ times higher).

The affinity was also evaluated in vivo, thanks to dorsal skinfold chamber studies performed after intravenous injection of NPs in mice bearing U87 xenografts tumors.AGuIX@ PS and AGuIX@PS@scramble NPs were located in the blood vessels at $1 \mathrm{~h}$ post injection but disappeared from them at $6 \mathrm{~h}$, due to the fenestration of the tumor vasculature. On the contrary, AGuIX@PS@KDKPPR particles were located onto the vessel walls of the tumor $1 \mathrm{~h}$ after intravenous injection and were still present $24 \mathrm{~h}$ after the administration. These qualitative fluorescence images support the important characteristics of our NPs; nevertheless, it will be helpful to complete them by quantitative data.AGuIX@PS@KDKPPR particles seem therefore really promising to detect the proliferating part of the tumor by MRI and in the context of VTP. The long-term targeting of the neovasculature seems an important asset for a further fractionated treatment.

\section{Conclusion}

In this study, we designed a new AGuIX NP for MRI-guided VTP of GBM. It combines MRI contrast agents, a PS for the photodynamic effect and a new peptide targeting NRP-1 receptor overexpressed by the tumor vasculature. It was shown that the photophysical properties of the PS and the peptide affinity for NRP-1 protein were preserved after the grafting onto AGuIX. The cellular uptake of NPs by HUVEC was increased twice compared to those without peptide or with a scramble peptide. The in vivo selectivity, evaluated using a skinfold chamber model in mice, confirmed that the KDKPPR-conjugated NPs were localized on the tumor vessel walls, highlighting their potential for VTP. Other experiments are in progress to fully describe the biodistribution of these NPs and to follow the VTP efficiency. T1-weighted imaging after intravenous injection of the targeted NPs will allow us to define the real tumor volume, taking into account the proliferating part of the tumor tissues. Besides, the drug-light interval will be optimized in real time according to the MRI signal intensity. Finally, the optimal irradiation scheme for iPDT will be developed as part of efficiency, inflammation and vascular responses.

\section{Acknowledgments}

This work was supported by the research funds of the French Ligue Nationale Contre le Cancer and EURONANOMED II "PhotoBrain" project (no ANR-14-ENM2-0001-01). The authors thankfully acknowledge Kevin Lemoine for his help in the synthesis of NPs. F Lux and O Tillement have 
one patent to disclose: WO2011135101. This patent protects some of the NPs described in this publication: AGuIX.

\section{Disclosure}

The authors have no other relevant affiliations or financial involvement with any organization or entity with a financial interest in or financial conflict with the subject matter or materials discussed in the manuscript apart from those disclosed. The authors report no other conflicts of interest in this work.

\section{References}

1. Munthe S, Sørensen MD, Thomassen M, et al. Migrating glioma cells express stem cell markers and give rise to new tumors upon xenografting. J Neurooncol. 2016;130(1):53-62.

2. Quirk BJ, Brandal G, Donlon S, et al. Photodynamic therapy (PDT) for malignant brain tumors - where do we stand? Photodiagnosis Photodyn Ther. 2015;12(3):530-544.

3. Hayashi K, Nakamura M, Miki H, et al. Photostable iodinated silica/ porphyrin hybrid nanoparticles with heavy-atom effect for wide-field photodynamic/photothermal therapy using single light source. $A d v$ Funct Mater. 2014;24(4):503-513.

4. Du K, Mick R, Busch TM, et al. Preliminary results of interstitial motexafin lutetium-mediated PDT for prostate cancer. Lasers Surg Med. 2006;38(5):427-434.

5. Swartling J, Höglund OV, Hansson K, Södersten F, Axelsson J, Lagerstedt A-S. Online dosimetry for temoporfin-mediated interstitia photodynamic therapy using the canine prostate as model. J Biomed Opt. 2016;21(2):028002-028002.

6. Eljamel S. Photodynamic applications in brain tumors: a comprehensive review of the literature. Photodiagnosis Photodyn Ther. 2010;7(2): 76-85.

7. Bechet D, Mordon SR, Guillemin F, Barberi-Heyob MA. Photodynamic therapy of malignant brain tumours: a complementary approach to conventional therapies. Cancer Treat Rev. 2014;40(2):229-241.

8. Kostron H. Photodynamic diagnosis and therapy and the brain. In: Gomer CJ, editor. Photodynamic Therapy. Methods in Molecular Biology. New York, NY: Humana Press; 2010:261-280.

9. Muragaki Y, Akimoto J, Maruyama T, et al. Phase II clinical study on intraoperative photodynamic therapy with talaporfin sodium and semiconductor laser in patients with malignant brain tumors. J Neurosurg. 2013; 119(4):845-852.

10. Lucky SS, Soo KC, Zhang Y. Nanoparticles in photodynamic therapy. Chem Rev. 2015;115(4):1990-2042

11. Couleaud P, Bechet D, Vanderesse R, et al. Functionalized silicabased nanoparticles for photodynamic therapy. Nanomedicine (Lond). 2011;6(6):995-1009.

12. Bechet D, Couleaud P, Frochot C, Viriot M-L, Guillemin F, BarberiHeyob M. Nanoparticles as vehicles for delivery of photodynamic therapy agents. Trends Biotechnol. 2008;26(11):612-621.

13. Wang S, Gao R, Zhou F, Selke M. Nanomaterials and singlet oxygen photosensitizers: potential applications in photodynamic therapy. J Mater Chem. 2004;14(4):487-493.

14. Koo Y-EL, Reddy GR, Bhojani M, et al. Brain cancer diagnosis and therapy with nanoplatforms. Adv Drug Deliv Rev. 2006;58(14): 1556-1577.

15. Lee D-E, Koo H, Sun I-C, Ryu JH, Kim K, Kwon IC. Multifunctional nanoparticles for multimodal imaging and theragnosis. Chem Soc Rev. 2012;41(7):2656-2672.

16. Gong H, Dong Z, Liu Y, et al. Engineering of multifunctional nanomicelles for combined photothermal and photodynamic therapy under the guidance of multimodal imaging. Adv Funct Mater. 2014;24(41) 6492-6502.
17. Liu Z-T, Xiong L, Liu Z-P, Miao X-Y, Lin L-W, Wen Y. In vivo and in vitro evaluation of the cytotoxic effects of Photosan-loaded hollow silica nanoparticles on liver cancer. Nanoscale Res Lett. 2014;9(1):1-8.

18. Li L, Nurunnabi M, Nafiujjaman M, Jeong YY, Lee Y, Huh KM. A photosensitizer-conjugated magnetic iron oxide/gold hybrid nanoparticle as an activatable platform for photodynamic cancer therapy. J Mater Chem B. 2014;2(19):2929.

19. Ismail AFM, Ali MM, Ismail LFM. Photodynamic therapy mediated antiproliferative activity of some metal-doped $\mathrm{ZnO}$ nanoparticles in human liver adenocarcinoma HepG2 cells under UV irradiation. J Photochem Photobiol B. 2014;138:99-108.

20. Yin M, Ju E, Chen Z, Li Z, Ren J, Qu X. Upconverting nanoparticles with a mesoporous $\mathrm{TiO}_{2}$ shell for near-infrared-triggered drug delivery and synergistic targeted cancer therapy. Chemistry. 2014;20(43): 14012-14017.

21. Secret E, Maynadier M, Gallud A, et al. Two-photon excitation of porphyrin-functionalized porous silicon nanoparticles for photodynamic therapy. Adv Mater. 2014;26(45):7643-7648.

22. Reddy GR, Bhojani MS, McConville P, et al. Vascular targeted nanoparticles for imaging and treatment of brain tumors. Clin Cancer Res. 2006;12(22):6677-6686.

23. Fan $\mathrm{W}, \mathrm{Bu} \mathrm{W}$, Shi J. On the latest three-stage development of nanomedicines based on upconversion nanoparticles. Adv Mater. 2016; 28(21):3987-4011.

24. Colombeau L, Acherar S, Baros F, et al. Inorganic nanoparticles for photodynamic therapy. Top Curr Chem. 2016;370:113-134.

25. Toussaint M, Pinel S, Auger F, et al. Proton MR spectroscopy and diffusion MRI imaging monitoring to predict tumor response to interstitial photodynamic therapy for glioblastoma. Theranostics. 2017;7(2): 436-451.

26. Tirand L, Frochot C, Vanderesse R, et al. A peptide competing with VEGF165 binding on neuropilin-1 mediates targeting of a chlorin-type photosensitizer and potentiates its photodynamic activity in human endothelial cells. J Control Release. 2006;111(1-2):153-164.

27. Zhang B, Wang H, Liao Z, et al. EGFP-EGF1-conjugated nanoparticles for targeting both neovascular and glioma cells in therapy of brain glioma. Biomaterials. 2014;35(13):4133-4145.

28. Tirand L, Bastogne T, Bechet D, et al. Response surface methodology: an extensive potential to optimize in vivo photodynamic therapy conditions. Int J Radiat Oncol Biol Phys. 2009;75(1):244-252.

29. Benachour H, Sève A, Bastogne T, et al. Multifunctional peptideconjugated hybrid silica nanoparticles for photodynamic therapy and MRI. Theranostics. 2012;2(9):889-904.

30. Graziani G, Lacal PM. Neuropilin-1 as therapeutic target for malignant melanoma. Front Oncol. 2015;5:125.

31. Thomas N, Bechet D, Becuwe P, et al. Peptide-conjugated chlorin-type photosensitizer binds neuropilin-1 in vitro and in vivo. $J$ Photochem Photobiol B. 2009;96(2):101-108.

32. Bechet D, Tirand L, Faivre B, et al. Neuropilin-1 targeting photosensitization-induced early stages of thrombosis via tissue factor release. Pharm Res. 2010;27(3):468-479.

33. Bechet D, Auger F, Couleaud P, et al. Multifunctional ultrasmall nanoplatforms for vascular-targeted interstitial photodynamic therapy of brain tumors guided by real-time MRI. Nanomedicine. 2015;11(3): $657-670$.

34. Kamarulzaman EE, Vanderesse R, Gazzali AM, et al. Molecular modelling, synthesis and biological evaluation of peptide inhibitors as anti-angiogenic agent targeting neuropilin-1 for anticancer application. J Biomol Struct Dyn. 2017;35(1):26-45.

35. Tylcz J-B, El Alaoui-Lasmaili K, Djermoune E-H, Thomas N, Faivre B, Bastogne T. Data-driven modeling and characterization of anti-angiogenic molecule effects on tumoral vascular density. Biomed Signal Process Control. 2015;20:52-60.

36. Le Duc G, Roux S, Paruta-Tuarez A, et al. Advantages of gadolinium based ultrasmall nanoparticles vs molecular gadolinium chelates for radiotherapy guided by MRI for glioma treatment. Cancer Nanotechnol. 2014;5(1):1. 
37. Stallivieri A, Colombeau L, Jetpisbayeva G, et al. Folic acid conjugates with photosensitizers for cancer targeting in photodynamic therapy: synthesis and photophysical properties. Bioorg Med Chem. 2017; 25(1):1-10.

38. Hermanson GT. Bioconjugate Techniques. Cambridge, MA: Academic Press; 2013.

39. Moser M, Behnke T, Hamers-Allin C, Klein-Hartwig K, Falkenhagen J, Resch-Genger U. Quantification of PEG-maleimide ligands and coupling efficiencies on nanoparticles with Ellman's reagent. Anal Chem. 2015;87(18):9376-9383.

40. Lim C-K, Heo J, Shin S, et al. Nanophotosensitizers toward advanced photodynamic therapy of cancer. Cancer Lett. 2013;334(2):176-187.

41. Schmitt F, Juillerat-Jeanneret L. Drug targeting strategies for photodynamic therapy. Anticancer Agents Med Chem. 2012;12(5):500-525.

42. Ichikawa K, Hikita T, Maeda N, et al. Antiangiogenic photodynamic therapy (PDT) by using long-circulating liposomes modified with peptide specific to angiogenic vessels. Biochim Biophys Acta. 2005;1669(1): 69-74.

43. Kopelman R, Lee Koo Y-E, Philbert M, et al. Multifunctional nanoparticle platforms for in vivo MRI enhancement and photodynamic therapy of a rat brain cancer. J Magn Magn Mater. 2005;293(1):404-410.
44. Kotb S, Detappe A, Lux F, et al. Gadolinium-based nanoparticles and radiation therapy for multiple brain melanoma metastases: proof of concept before phase I trial. Theranostics. 2016;6(3):418-427.

45. Bianchi A, Moncelet D, Lux F, et al. Orotracheal administration of contrast agents: a new protocol for brain tumor targeting: a new protocol to target brain cancer. NMR Biomed. 2015;28(6):738-746.

46. Sancey L, Kotb S, Truillet C, et al. Long-term in vivo clearance of gadolinium-based AGuIX nanoparticles and their biocompatibility after systemic injection. ACS Nano. 2015;9(3):2477-2488.

47. Mignot A, Truillet C, Lux F, et al. A top-down synthesis route to ultrasmall multifunctional Gd-based silica nanoparticles for theranostic applications. Chemistry. 2013;19(19):6122-6136.

48. Lux F, Mignot A, Mowat P, et al. Ultrasmall rigid particles as multimodal probes for medical applications. Angew Chem Int Ed. 2011;50(51): 12299-12303.

49. Chu Z, Zhang S, Yin C, Lin G, Li Q. Designing nanoparticle carriers for enhanced drug efficacy in photodynamic therapy. Biomater Sci. 2014;2(6):827-832.

50. Tang W, Xu H, Kopelman R, Philbert MA. Photodynamic characterization and in vitro application of methylene blue-containing nanoparticle platforms. Photochem Photobiol. 2005;81(2):242-249.
International Journal of Nanomedicine

\section{Publish your work in this journal}

The International Journal of Nanomedicine is an international, peerreviewed journal focusing on the application of nanotechnology in diagnostics, therapeutics, and drug delivery systems throughout the biomedical field. This journal is indexed on PubMed Central, MedLine, CAS, SciSearch ${ }^{\circledR}$, Current Contents ${ }^{\circledR} /$ Clinical Medicine,

\section{Dovepress}

Journal Citation Reports/Science Edition, EMBase, Scopus and the Elsevier Bibliographic databases. The manuscript management system is completely online and includes a very quick and fair peer-review system, which is all easy to use. Visit http://www.dovepress.com/ testimonials.php to read real quotes from published authors. 